\title{
Transients that may affect low voltage electrical systems
}

\begin{abstract}
A comprehensive review has been done on the types of transients that may affect low voltage electrical systems. The paper discusses various characteristics of lightning, switching, nuclear and intentional microwave impulses giving special attention to their impact on equipment and systems. The analysis shows that transients have a wide range of rise time, half peak width, action integral etc. with respect to both source and coupling mechanism. Hence, transient protection technology should be more specific with regard to the capabilities of the protection devices.
\end{abstract}

